\title{
Modeling Sporulation of Fusicladium carpophilum on Nectarine Twig Lesions: Relative Humidity and Temperature Effects
}

\author{
N. Lalancette, K. A. McFarland, and A. L. Burnett \\ Rutgers University, Department of Plant Biology and Pathology, Rutgers Agricultural Research and Extension Center, 121 Northville Road, \\ Bridgeton, NJ 08302-5919. \\ Current address of A. L. Burnett: FMC Corporation, 701 Princeton South Corporate Center, Ewing, NJ 08628. \\ Accepted for publication 16 November 2011.
}

\begin{abstract}
Lalancette, N., McFarland, K. A., and Burnett, A. L. 2012. Modeling sporulation of Fusicladium carpophilum on nectarine twig lesions: Relative humidity and temperature effects. Phytopathology 102:421-428.

The production of conidia by Fusicladium carpophilum on twig lesions was quantitatively modeled as a function of temperature and duration of high relative humidity. During peak sporulation periods in 2007, 2008, and 2009, 1-year-old twigs bearing abundant overwintering lesions were removed from a heavily infected 'Redgold' nectarine orchard, placed in trays at high relative humidity ( $>95 \%)$, and incubated at eight constant temperatures for seven durations, resulting in a factorial design of 56 treatment combinations. Conidia numbers were estimated with a hemacytometer. Results from a six-stage modeling process indicated that, at any given temperature, spore production during high relative humidity periods

increased in a monomolecular- to Gompertz-like pattern. The Richards model, with shape parameters of 0.79 to 0.90 , was found to provide the best overall fit. When the asymptote and rate parameters were derived as functions of temperature using Gaussian and quadratic models, respectively, the duration of high relative humidity and temperature described 90 to $94 \%$ of the variation in conidia production. Predictions of the final models were highly correlated with observed levels of sporulation $(r>$ 0.94; $P<0.0001$ ), indicating an excellent fit to the data. The optimum temperature for sporulation, based on fitting a Gaussian model to the maximum sporulation levels at each temperature, was 17.9 to $20.2^{\circ} \mathrm{C}$, with an overall average of $18.8^{\circ} \mathrm{C}$. The derived models give a quantitative description of sporulation by $F$. carpophilum and may have potential use in simulators and forecasting systems.
\end{abstract}

Peach and nectarine scab is caused by the plant-pathogenic fungus Fusicladium carpophilum (Thüm.) Oudem. (syn. Cladosporium carpophilum and Fusicladosporium carpophilum) (26). The disease occurs wherever these crops are grown in warm, humid climates accompanied by frequent rainfall $(1,7)$. Thus, in the United States, scab is most prevalent and problematic in the eastern half of the country. The role of the teleomorph stage Venturia carpophila in the disease cycle and epidemiology of peach scab is unknown.

Fusicladium carpophilum overwinters as mycelia in lesions on fruit-bearing 1-year-old twigs $(7,10)$. Conidial production on these lesions commences at bloom in spring and continues for $\approx 10$ weeks, tapering off during early to mid-summer $(5,19,22,29)$. These asexual spores, which are produced on olive-colored tufts of conidiophores, act as the primary inoculum for the ensuing epidemic. Conidia are disseminated during rainy periods to newly formed fruit, current season vegetative shoots, and foliage (10). Recent research on inoculum dispersal mechanisms demonstrated that fruit infection results primarily from rain splash and twig runoff (18).

New lesions appear on current-season twigs at approximately the same time as disease is first observed on fruit. Most of these lesions do not completely develop and become infectious until the following year $(10,19)$. Some new twig lesions may produce conidia later in the season but this inoculum is of little importance

Corresponding author: N. Lalancette; E-mail address: lalancette@ njaes.rutgers.edu

* The $\boldsymbol{e}$-Xtra logo stands for "electronic extra" and indicates that the online version contains two supplementary tables and four supplementary figures.

http://dx.doi.org/10.1094/PHYTO-08-10-0222

(c) 2012 The American Phytopathological Society in the disease cycle (1). Foliar infections are not common, and produce few conidia later in the season. In general, secondary inoculum from fruit infections also does not contribute significantly to epidemic development because the latent period is quite long, 42 to 77 days (7). Such secondary cycles would be most important on late cultivars that sustained early fruit infections. Thus, the majority of inoculum for the current-season epidemic originates from overwintering lesions on 1-year-old twigs. The pathogen does not survive on 2-year-old or older wood.

Given the above, twig lesions are an important inoculum source for both the initiation and propagation of scab epidemics. Thus, quantitative information on the effects of environmental factors on sporulation of twig lesions is critical to understanding the epidemiology of peach scab. Research investigating the relationship between atmospheric moisture and spore production by $F$. carpophilum has been conducted (19). Results showed that twig lesion sporulation was induced by periods of high relative humidity (RH). When incubated at a constant $25^{\circ} \mathrm{C}$, twig lesion sporulation only occurred at 80 to $100 \% \mathrm{RH}$ and was most abundant at near-saturation values of 98 to $100 \% \mathrm{RH}$.

Sporulation by a fungal plant pathogen consists of three sequential phases: sporophore production, spore production, and spore maturation (8). We propose that this sporulation process for $F$. carpophilum is primarily dependent on the occurrence of favorable temperatures and durations of high RH. Currently, no data are available that describe the combined effects of moisture and temperature over time on production of conidia on twig lesions. In particular, the optimum temperatures and durations of high RH, as well as the extremes of these factors that limit sporulation, are not known. Thus, the main objective of this study was to quantitatively describe the sporulation process of $F$. carpophilum over the full range of these two environmental factors. Because the effects of these two partially independent variables 
are examined simultaneously, mathematical models depicting a response surface were developed to provide a visualization of their interaction as well as to generate estimates of descriptive parameters. Partial results of this study were reported previously (17).

\section{MATERIALS AND METHODS}

Twig samples. During the peak sporulation periods in 2007, 2008 , and 2009, twigs bearing abundant overwintering scab lesions were pruned from trees in a mature, heavily infected nectarine orchard (Prunus persica var. nucipersica 'Redgold'). The orchard, located at the Rutgers Agricultural Research and Extension Center (RAREC), Bridgeton, NJ, had not received any fungicide applications during this period. Twigs were cut to a length of $15 \mathrm{~cm}$, measured from the distal growing tip backward. After leaf removal, they were washed in running tap water for $30 \mathrm{~s}$ to remove debris and darken the scab lesions for easier examination. The total number of lesions on each twig was then counted using a dissecting microscope at low power. The length of each twig was scanned three times, rotating the twig by onethird between scans; the green underside of the twigs served as an initial reference point. Based on these counts, twigs were arranged in groups of 10, such that each group (sample) had approximately the same number of lesions. Finally, twig samples were placed in sealed plastic bags and put in a refrigerator overnight at $5^{\circ} \mathrm{C}$.

Incubation treatments. At the beginning of day 2, the 10-twig samples were removed from the refrigerator and placed in a flask containing 1 liter of deionized water at $5^{\circ} \mathrm{C}$ plus 1 drop of Tween 20. After shaking the flask vigorously for $1 \mathrm{~min}$ to remove any spores previously formed, the twigs were rinsed in deionized water and then patted dry with paper towels. The 10-twig samples were then placed in plastic trays (18 by 14 by $3 \mathrm{~cm}$ deep) maintained at high $(>95 \%) \mathrm{RH}$ by lining the bottom with moist paper towel and covering the tray with a tight-fitting lid. Twigs were placed across two 8-mm-diameter plastic tubes to elevate them above the moistened paper towel. To further ensure a high humidity level throughout the tray volume, the sides and bottom of the lid were atomized with deionized water to produce a fine layer of water beads. Trays were then placed in an incubator for a specified duration at a designated constant temperature. The lid was installed after a 1- to 2-min delay to allow tray air volume to more quickly equilibrate to incubator temperatures. Water condensation on the clear plastic sides of the trays was monitored to ensure maintenance of a near-saturated atmosphere within the tray.

Incubation treatments consisted of an eight-by-seven factorial of temperature and duration of high $\mathrm{RH}$. Temperature levels were $1,5,10,15,20,25,30$, and $35^{\circ} \mathrm{C}$; incubation durations were 4,8 , 12, 24, 36, 48, and $72 \mathrm{~h}$. Thus, in each year, 56 treatment combinations were examined utilizing 560 twigs. The order of examination of each treatment temperature level within each yearly repetition was chosen randomly.

Temperatures during incubation periods were recorded electronically using portable Hobo data loggers placed on the same incubator shelf adjacent to the trays (Onset Computer Corp., Bourne, MA). Accuracy of these instruments was checked at three temperature points $\left(5,20\right.$, and $\left.35^{\circ} \mathrm{C}\right)$ using a National Institute of Standards and Technology-certified mercury thermometer accurate to $0.1^{\circ} \mathrm{C}$. Any temperature deviations from this standard, which were 0.1 to $0.5^{\circ} \mathrm{C}$, were corrected based on the generated calibration curves.

Sporulation assessment. After completion of the designated incubation period, twigs from each sample were individually spray washed with water eight times (twice per quarter turn) using a DeVilbiss atomizer set at $52 \mathrm{kPa}$ (DeVilbiss Health Care, Inc., Somerset, PA). Conidia from each 10-twig sample were bulk collected in a flask; total volumes were 30 to $40 \mathrm{ml} / \mathrm{sample}$. After agitation, the concentration of conidia in the suspension was estimated by averaging 10 hemacytometer chamber counts performed with the aid of a compound microscope $(\times 200)$. Total number of conidia produced per sample was determined from this concentration and total volume of the suspension. From these recorded data and the earlier lesion counts, two dependent variables were calculated for each treatment combination of temperature and duration of high RH: $S_{1}=$ number of conidia per lesion and $S_{2}=$ number of conidia per centimeter of twig length = number of conidia $/ 150 \mathrm{~cm}$. As a result of sample pooling, no estimates of within-year variation could be made.

Model development. The procedure used for modeling sporulation as a function of temperature and high $\mathrm{RH}$ duration consisted of six interrelated steps. First, the upper asymptote of each sporulation-dependent variable needed to be estimated from the data. This task was accomplished by searching for the maximum mean value across all durations of incubation within each temperature treatment. These means served as constants when estimating other parameters during subsequent intermediate operations.

In the second step, the Richards model, chosen for its flexibility in conforming to different patterns of growth (28), was fit to data from each year as well as the combined data (all years):

$$
\begin{aligned}
& \mathrm{S}=K\left(1-B e^{-r \mathrm{H}}\right)^{1 /(1-m)} \text { for } m<1 \\
& \mathrm{~S}=K\left(1+B e^{-r \mathrm{H}}\right)^{1 /(1-m)} \text { for } m>1
\end{aligned}
$$

in which $\mathrm{S}=$ dependent sporulation variable $\left(\mathrm{S}_{1}\right.$ or $\left.\mathrm{S}_{2}\right), K=$ estimated asymptote from step $1, m=$ shape parameter, $r=$ rate parameter, and $\mathrm{H}=$ duration of $\mathrm{RH}>95 \%$. The constants of integration, $B$, were $1-\left(\mathrm{S}_{0} / K\right)^{1-m}$ and $\left(\mathrm{S}_{0} / K\right)^{1-m}-1$ for equations 1 and 2 , respectively. The initial sporulation level $\left(\mathrm{S}_{0}\right)$ was set equal to 0.01 because little or no conidia were assumed present at initiation of incubation. These regression analyses provided overall estimates of the shape parameter for each year's data as well as for the combined data.

In the third step, the linearized version of the Richards model was fit to the data for each temperature:

$$
\begin{array}{ll}
\ln \left\{1 /\left[1-(\mathrm{S} / K)^{1-m}\right]\right\}=r \mathrm{H} & \text { for } m<1 \\
\ln \left\{1 /\left[(\mathrm{S} / \mathrm{K})^{1-m}-1\right]\right\}=r \mathrm{H} & \text { for } m>1
\end{array}
$$

in which $\mathrm{S}=$ dependent sporulation variable $\left(\mathrm{S}_{1}\right.$ or $\left.\mathrm{S}_{2}\right), K=$ estimated asymptote from step $1, m=$ estimated shape parameter from step 2, $r=$ rate parameter, and $\mathrm{H}=$ duration of $\mathrm{RH}>95 \%$. A no-intercept version was fit because zero sporulation occurred at $\mathrm{H}=0 \mathrm{~h}$ (i.e., no conidia were assumed present at initiation of incubation). To avoid division by zero, the constant 0.01 was added to $K$. Separate models were fit to data at each temperature within each year as well as across all 3 years (combined).

In the fourth development step, the rate parameter estimates from equation 3 or 4 were plotted against temperature for each combination of dependent variable and experimental replication (year). Visual examination of these graphs indicated a parabolalike relationship between rate and temperature; no inflections were observed at upper or lower ends of the temperature range examined and, thus, the relationship between the rate parameter and temperature $(\mathrm{T})$ was modeled using a quadratic function:

$$
r=b_{0}+b_{1} \mathrm{~T}+b_{2} \mathrm{~T}^{2}
$$

where $r=$ Richards model rate parameter estimates $r_{1}$ and $r_{1}$ for each dependent variable $S_{1}$ and $S_{2}$, respectively.

In the fifth step, the two sporulation-dependent variables were modeled as functions of $\mathrm{T}$ and $\mathrm{H}$ by substituting the rate param- 
eter function (equation 5) for $r$ in the nonlinear form of the Richards model (equation 1 or 2) to yield:

$$
\begin{array}{ll}
\mathrm{S}=K\left(1-B e^{-b_{0} \mathrm{H}-b_{1} \mathrm{HT}-b_{2} \mathrm{HT}^{2}}\right)^{1 /(1-\mathrm{m})} & \text { for } m<1 \\
\mathrm{~S}=K\left(1+B e^{-b_{0} \mathrm{H}-b_{1} \mathrm{HT}-b_{2} \mathrm{HT}^{2}}\right)^{1 /(1-\mathrm{m})} & \text { for } m>1
\end{array}
$$

The shape parameter $m$ and $b_{0}, b_{1}$, and $b_{2}$ coefficients were estimated for the combined data set (all years). As in equations 1 and 2, $K$ was set equal to the asymptote values derived in step 1 and $\mathrm{S}_{0}$ was set equal to 0.01 in the constants of integration $(B)$.

The final step in the development process consisted of modeling the upper asymptote $K$ as a function of temperature T. This step was necessary because $K$, at each temperature level, is a constant in equations 6 and 7. To visualize the nature of this relationship, the maximum mean sporulation values estimated in step 1 were plotted against their respective temperatures. The resulting curves had shapes resembling that of a normal (Gaussian) distribution with optimum, maximum, and minimum values, as well as slight inflections as temperatures approached each extreme. Thus, the relationship between $K$ and $\mathrm{T}$ was modeled by fitting a generalized form of the Gaussian function:

$$
K=a \exp \left[-s(\mathrm{~T}-t)^{2}\right]
$$

where $a, s$, and $t$ describe the curve's amplitude, spread, and location of its maximum, respectively. In terms of pathogen biology, $a$ is the maximum amount of sporulation, $s$ determines the temperature range over which sporulation occurs (spread between the minimum and maximum temperatures), and $t$ is the optimum temperature for sporulation. Together, this equation, along with equation 6 or 7 , constitutes the final model for predicting sporulation as a function of temperature and duration of high RH. One set of equations 6 or 7 and 8 was created for each dependent variable.

Model validation. To examine the ability of the final model to accurately and precisely predict sporulation, a validation model was created by fitting the multidimensional Richards function (equation 6 or 7 ) to a majority subset of the data. This validation data set, which consisted of 132 observations or $\approx 80 \%$ of the collected data, was created by randomly choosing 44 observations from the 56 observations recorded in each year. The remaining 36 observations, 12 from each year, were used as test data. Because not all maximum sporulation values were randomly chosen to be part of the validation data set, a Gaussian validation model (equation 8) was also created for estimating $K$.

Predicted sporulation values from the combined Richards and Gaussian validation models were plotted against the observed test data values. A simple linear regression was performed to evaluate the degree of association between the observed and predicted values. Furthermore, $t$ tests were performed to compare the slope and intercept of the derived line to one and zero, respectively.

Statistical analysis. The Richards models in the second and fifth steps (equations 1, 2, 6, and 7) and the Gaussian model in the final step (equation 8) of the model development process were fit using the nonlinear regression (NLIN) procedure of the Statistical Analysis System (v9.2; SAS Institute, Cary, NC). The GaussNewton iterative method was used to solve the nonlinear leastsquares. The linearized forms of the Richards model in step 3 (equations 3 and 4) and the quadratic model in step four (equation 5) were fit using the SAS linear regression (REG) procedure. All regression analyses were performed on the data values derived from the 10-twig samples; that is, within each replicate year, there was one observation per combination of temperature and duration of high RH.

Goodness-of-fit of linear model equations 3, 4, and 5 was evaluated by examination of the coefficients of determination, the standard errors of the parameter estimates, and standardized residual plots (23). Aptness of nonlinear equations 1, 2, 6, 7, and 8 was ascertained by examining the coefficients of variation of the parameter estimates and by examining graphs of the standardized residuals plotted against predicted values.

Statistical procedures used to derive the corresponding validation models and to assess their fit were identical to that used for analysis of the complete data set.

\section{RESULTS}

Treatment effects. In each year of the study, sporulation was observed at 5 to $30^{\circ} \mathrm{C}$, with very low levels of conidia production at the temperature extremes of 1 and $35^{\circ} \mathrm{C}$ (Fig. 1; Supplementary Figure 1). Maximum sporulation in 2007 and 2009 occurred after $72 \mathrm{~h}$ of incubation at $15^{\circ} \mathrm{C}$ whereas, in 2008 , the maximum was observed after $72 \mathrm{~h}$ at $20^{\circ} \mathrm{C}$. Overall levels of spore production
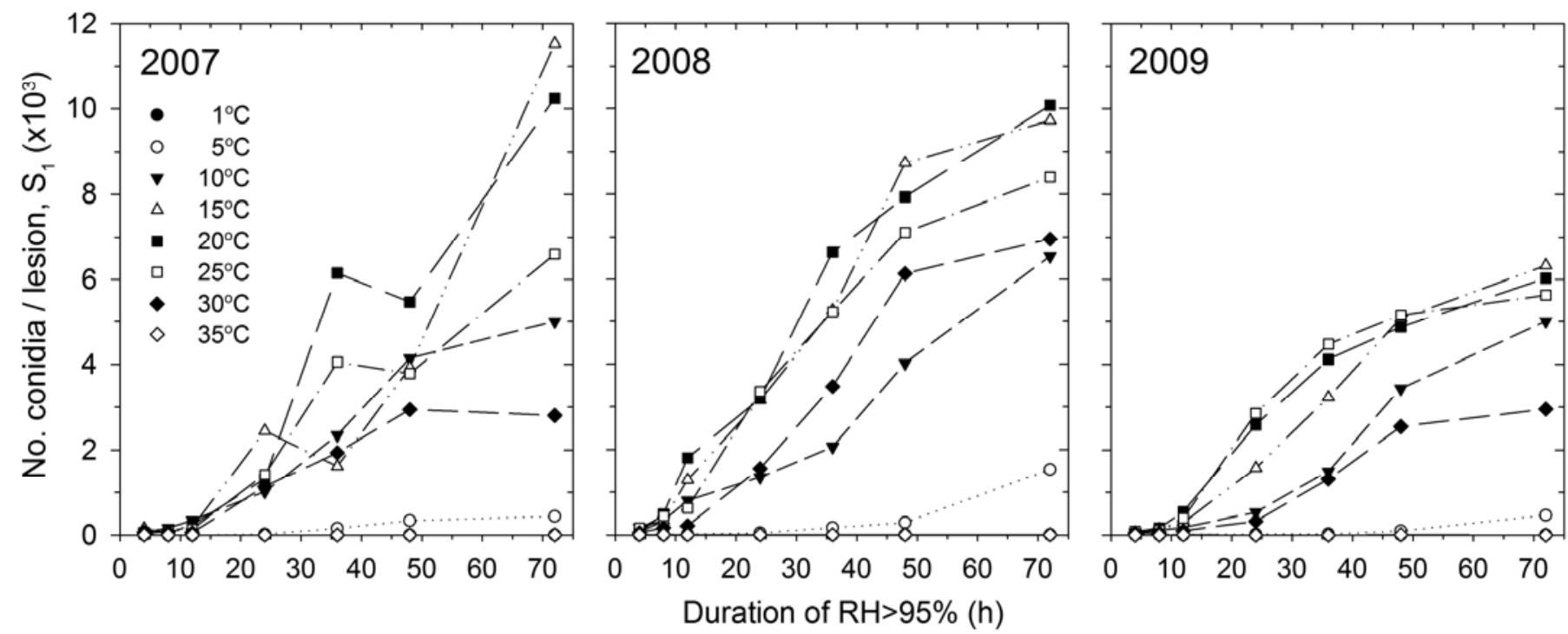

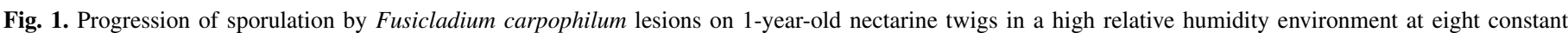

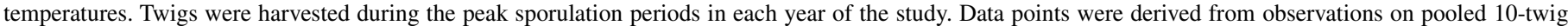

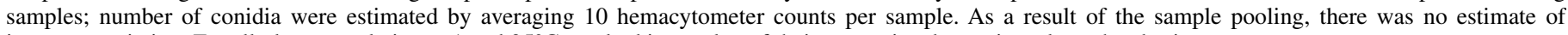
intrayear variation. Equally low sporulation at 1 and $35^{\circ} \mathrm{C}$ resulted in overlap of their respective data points along the abscissa. 
were fairly similar in 2007 and 2008, attaining maximums of $11.53 \times 10^{3}$ and $10.07 \times 10^{3}$ conidia/lesion, respectively. In contrast, the maximum recorded in $2009,6.32 \times 10^{3}$ conidia/lesion, was 38 to $45 \%$ lower than observed in these earlier years. Similar patterns of sporulation maxima were observed for the number of conidia per twig length dependent variable. The 2007 and 2008 maxima were $7.52 \times 10^{4}$ and $6.74 \times 10^{4}$ conidia/cm, respectively, while the 2009 maximum was 26 to $34 \%$ lower at $4.97 \times 10^{4}$ conidia/cm.
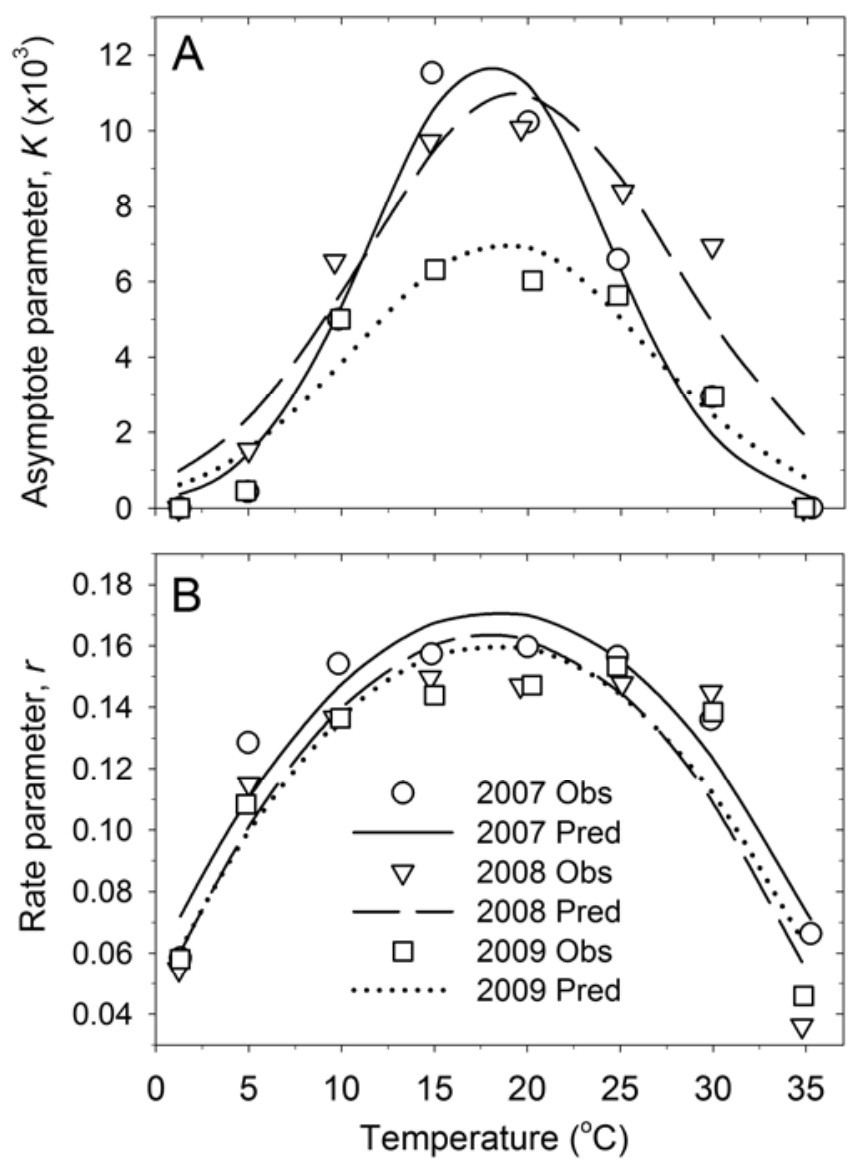

Fig. 2. Influence of temperature on the $\mathbf{A}$, asymptote parameter $K$ and $\mathbf{B}$, rate parameter $r$ of the Richards model for the number of conidia per lesion $\left(\mathrm{S}_{1}\right)$ dependent variable describing sporulation of Fusicladium carpophilum on nectarine twig lesions. Data points for the asymptote parameter were maximum sporulation values observed across all durations of incubation at each temperature; lines represent predicted values of the Gaussian model (equation 8). Data points for the rate parameter were estimated by fitting the Richards model (equation 3) to sporulation curves at each temperature level; lines represent predicted values of the quadratic model (equation 5).
The shapes of the sporulation curves for most of the temperature levels were somewhat linear in appearance between 0 and 50 $\mathrm{h}$ duration of high RH (Fig. 1). Some curves showed an initial slow rate of spore production, resulting in a slight upward curvature at early durations. After $50 \mathrm{~h}$, the rate of increase slowed for most curves as they approached $72 \mathrm{~h}$. A greater degree of withintemperature treatment variability in 2007 made it difficult to discern the shape of the sporulation curves in that year.

The number of lesions per twig in harvested samples varied across the 3 years of the study. The mean number of lesions per twig ( $\pm 95 \%$ confidence interval), calculated across all treatment combinations in each year, was $95.6 \pm 3.5$ in $2007,72.3 \pm 2.8$ in 2008 , and $104.7 \pm 3.3$ in 2009 . Thus, lesion numbers in 2009 , on average, were $9.5 \%$ greater than observed in 2007 and $45 \%$ higher than in 2008. Median values of 89,66 , and 100.5 lesions/ twig for 2007, 2008, and 2009, respectively, also indicated that a greater proportion of twigs had higher lesion counts in 2009.

Estimation of $\boldsymbol{K}$. The upper asymptote $K$, estimated by the maximum mean value of observed sporulation $\left(S_{1}\right.$ and $\left.S_{2}\right)$, was attained after $72 \mathrm{~h}$ of incubation for most temperature levels (Fig 1 ). The one exception occurred at $30^{\circ} \mathrm{C}$ in 2007 , when the maximum occurred at the 48-h assessment. When the asymptotes were fit as a function of temperature via the Gaussian model, graphical examination of the observed and predicted values showed a reasonably good fit of the model to the data for each year of the study (Fig. 2A; Supplementary Figure 2A). Overall, the fit of the model appeared somewhat better for the $\mathrm{S}_{2}$-dependent variable, especially at higher temperatures $\left(\geq 30^{\circ} \mathrm{C}\right)$ for the 2008 data. Results of the nonlinear regression analyses, which agree with this visual observation, showed that the total coefficient of variation $(\mathrm{CV})$ for the parameter estimates, $\mathrm{CV}_{\mathrm{T}}$, was $13.5 \%$ higher for $S_{1}$ than $S_{2}$ in 2008 (Table 1; Supplementary Table 1). Nevertheless, most individual parameter $\mathrm{CV}$ values were quite low across the 3 years: 2.6 to $23.7 \%$ for $S_{1}$ and 2.7 to $24.4 \%$ for $\mathrm{S}_{2}$.

Coefficient estimates across the 3 years were more variable for the amplitude $(a)$ and spread $(s)$ parameters than for the optimum temperature $(t)$ parameter (Table 1). Furthermore, these differences among years were greater for $S_{1}$ than for $S_{2}$. For example, the $2009 \mathrm{~S}_{1}$ estimate for the amplitude parameter was 56 and $68 \%$ lower than the 2008 and 2007 estimates, respectively. In comparison, the 2009 amplitude estimate for $S_{2}$ was $18 \%$ lower than the 2008 estimate and 37\% lower than the 2007 estimate. These differences in amplitude were also quite evident in the graphs (Fig. 2A). Similar differences were observed for the spread parameter estimates. In contrast, estimates of optimum temperatures for sporulation $(s)$ varied across all 3 years by only $1.5^{\circ} \mathrm{C}$ for $\mathrm{S}_{1}$ and $2.2^{\circ} \mathrm{C}$ for $\mathrm{S}_{2}$, a difference of only 8.5 and $12.5 \%$ between the minimum and maximum estimates, respectively.

Estimation of $\boldsymbol{m}$. Although final values of the shape parameter were estimated for equations 6 and 7 in the fifth step of the model

TABLE 1. Estimation of the Richards model asymptote parameter $K$ by fitting the Gaussian model to maximum sporulation of Fusicladium carpophilum lesions on nectarine twigs observed at eight temperature levels ${ }^{\mathrm{a}}$

\begin{tabular}{|c|c|c|c|c|c|c|c|c|c|}
\hline \multirow[b]{2}{*}{ Model $^{\mathrm{b}}$} & \multicolumn{2}{|c|}{ Error } & \multicolumn{3}{|c|}{ Parameter estimate ${ }^{c}$} & \multicolumn{4}{|c|}{ Parameter CV $(\%)^{\mathrm{d}}$} \\
\hline & $\mathrm{df}$ & $\mathrm{MS}^{\mathrm{e}}$ & $a$ & $\mathrm{~s}$ & $t$ & $a$ & $\mathrm{~s}$ & $t$ & $\mathrm{CV}_{\mathrm{T}}$ \\
\hline 2007 & 5 & $8.38 \mathrm{E} 05$ & $1.18 \mathrm{E} 04$ & $1.26 \mathrm{E}-2$ & 17.93 & 6.4 & 14.6 & 2.6 & 23.6 \\
\hline 2008 & 5 & $2.36 \mathrm{E} 06$ & $1.10 \mathrm{E} 04$ & $7.31 \mathrm{E}-3$ & 19.44 & 10.2 & 23.3 & 4.9 & 38.4 \\
\hline Combined & 21 & $2.78 \mathrm{E} 06$ & $9.76 \mathrm{E} 03$ & $8.82 \mathrm{E}-3$ & 18.64 & 7.4 & 16.9 & 3.4 & 27.7 \\
\hline Validation & 18 & $2.28 \mathrm{E} 06$ & $1.05 \mathrm{E} 04$ & $9.19 \mathrm{E}-3$ & 18.79 & 7.2 & 15.3 & 3.0 & 25.5 \\
\hline
\end{tabular}

${ }^{a}$ Maximum sporulation $=$ number of conidia per lesion $\left(S_{1}\right)$; data values were calculated from pooled observations on 10 twigs per temperature level.

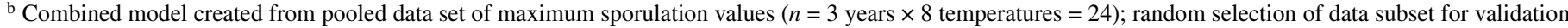
resulted in three missing maximum sporulation values; hence, $n=21$ for validation model.

${ }^{c}$ Gaussian model (equation 8) fit to each data set using nonlinear regression; all model $F$ values were highly significant $(P<0.0001)$.

${ }^{\mathrm{d}}$ Parameter coefficient of variation $(\mathrm{CV})=($ standard error/estimate $) \times 100 ; \mathrm{CV}_{\mathrm{T}}=$ total or sum of variation across all parameters.

e Scientific E notation (e.g., E05 $=\times 10^{5}$ and E-3 $=\times 10^{-3}$ ). 
development process, intermediate values of $m$ were estimated in step 2 to allow for estimation of the rate in step 3. For the $S_{1}$ variable, these $m$ values were $0.90,0.80,0.83,0.85$, and 0.90 for fitting the 2007, 2008, 2009, combined, and validation data sets, respectively. For the $\mathrm{S}_{2}$ variable, the $m$ values were $0.87,0.79$, $0.81,0.83$, and 0.87 for fitting the 2007, 2008, 2009, combined, and validation data sets, respectively. These values of $m$ describe a function with properties intermediate between a monomolecular model $(m=0)$ and a Gompertz model $(m=1)$. All model $F$ values from these nonlinear regression analyses were highly significant $(P<0.0001)$; coefficients of variation for the shape parameters were 2.5 to $7.2 \%$.

Estimation of $\boldsymbol{r}$. When the linearized Richards model was fit to the transformed sporulation data at each temperature (equation 3), mean $r^{2}$ values of $0.78,0.70$, and 0.72 for $S_{1}$ and $0.73,0.67$, and 0.70 for $S_{2}$ were observed in 2007, 2008, and 2009, respectively. The individual coefficients of determination were 0.67 to 0.87 for $\mathrm{S}_{1}$ and 0.61 to 0.83 for $\mathrm{S}_{2}$.

The quadratic model (equation 5) provided a reasonably good fit to the Richards model rate parameters derived at each temperature level (Fig. 2B). Across all 3 years of the study, the quadratic function of temperature described 79 to $89 \%$ of the variation in the rate parameter for the $S_{1}$-dependent variable and 81 to $91 \%$ of the variation for the $S_{2}$-dependent variable (Table 2; Supplementary Table 2). Standard errors for the $b_{1}$ and $b_{2}$ quadratic model parameters were 11.9 to $19.6 \%$ of their estimated values; standard errors for the $b_{0}$ parameter where higher, 17.7 to $45.2 \%$. Although these statistics were good overall, the model tended to underpredict the observed rate at $30^{\circ} \mathrm{C}$ and overpredict the rate at $35^{\circ} \mathrm{C}$ (Fig. 2B). These deviations were particularly evident for the 2008 and 2009 data.

Sporulation models. When the final regression model (equation 6) was fit to each dependent variable, model $F$ values were highly significant $(P<0.0001)$. Coefficients of variation for the parameter estimates were fairly low, $\approx 2$ to $30 \%$ (Table 3). Overall, the fit was somewhat better for the $\mathrm{S}_{1}$-dependent variable, with total parameter variation $\left(\mathrm{CV}_{\mathrm{T}}\right) \mathbf{1 6 . 5 \%}$ lower than obtained for $\mathrm{S}_{2}$. However, for both variables, standardized residuals were uniformly scattered about the zero line in the residual plots; furthermore, 91.1 and $92.9 \%$ of the standardized residuals were between -2 and +2 for $S_{1}$ and $S_{2}$, respectively.

The final nonlinear model describing twig lesion sporulation as an expression of the independent variables temperature and duration of high $\mathrm{RH}$ is

$$
\mathrm{S}=K\left(1-B e^{-b_{0} \mathrm{H}-b_{1} \mathrm{HT}-b_{2} \mathrm{HT}^{2}}\right)^{1 /(1-\mathrm{m})}
$$

where $\mathrm{S}$ is number of conidia per lesion $\left(\mathrm{S}_{1}\right)$ or number of conidia per centimeter of twig $\left(\mathrm{S}_{2}\right)$; $\mathrm{T}$ is air temperature $\left(1^{\circ} \mathrm{C} \leq \mathrm{T} \leq 35^{\circ} \mathrm{C}\right)$; $\mathrm{H}$ is hours of $\mathrm{RH}>95 \%(0 \leq \mathrm{H} \leq 72) ; K=a \exp \left[-s(\mathrm{~T}-t)^{2}\right]$ with parameters defined in Table $1 ; m, b_{0}, b_{1}$, and $b_{2}$ are defined in Table 3; and $B=1-(0.01 / K)^{1-\mathrm{m}}$. If solved for various values of T and $\mathrm{H}$, these equations describe a three-dimensional response surface depicting sporulation (Fig. 3; Supplementary Figure 3). To evaluate this model, predicted values of each dependent variable were generated from the independent variable data using parameters derived from the combined data set. These predictions were highly correlated with observed levels of sporulation (Fig. $3)$. Linear regression analysis of the predicted and observed data showed that temperature and duration of high $\mathrm{RH}$, as independent variables for the predictions, described 90 and $94 \%$ of the variation in the observed number of conidia per lesion and number of conidia per centimeter of twig, respectively.

Model validation. For each dependent variable, estimated parameter values for the Richards validation model (equation 6) were fairly similar to those estimated when fitting the model to the complete, combined data set (Table 3). The greatest difference occurred for the $b$ parameter, which was $\approx 18$ to $22 \%$ higher in

TABLE 2. Estimation of the Richards model rate parameter $r$ describing sporulation of Fusicladium carpophilum lesions on nectarine twigs as quadratic functions of temperature

\begin{tabular}{|c|c|c|c|c|c|c|c|c|c|}
\hline \multirow[b]{2}{*}{ Model $^{\mathrm{b}}$} & \multicolumn{2}{|c|}{ Error } & \multicolumn{3}{|c|}{ Parameter estimate $^{c}$} & \multicolumn{3}{|c|}{ Parameter standard error } & \multirow[b]{2}{*}{$R_{\mathrm{a}}^{2 \mathrm{e}}$} \\
\hline & $\mathrm{df}$ & $\mathrm{MS}^{\mathrm{d}}$ & $b_{0}$ & $b_{1}$ & $b_{2}$ & $b_{0}$ & $b_{1}$ & $b_{2}$ & \\
\hline 2007 & 5 & $1.87 \mathrm{E}-4$ & 0.0560 & 0.0126 & $-3.46 \mathrm{E}-4$ & 0.0127 & $1.68 \mathrm{E}-3$ & $4.49 \mathrm{E}-5$ & 0.89 \\
\hline Combined & 21 & $2.31 \mathrm{E}-4$ & 0.0479 & 0.0129 & $-3.57 \mathrm{E}-4$ & $8.12 \mathrm{E}-3$ & $1.08 \mathrm{E}-3$ & $2.92 \mathrm{E}-5$ & 0.87 \\
\hline
\end{tabular}

a Data consisted of $r$ parameter estimates generated from fitting the Richards model to number of conidia per lesion $\left(\mathrm{S}_{1}\right)$ at each temperature level (equation 3 ).

${ }^{\mathrm{b}}$ Combined model created from pooled data set $(n=3$ years $\times 8$ temperatures $=24)$.

${ }^{c}$ Quadratic model (equation 5) fit to each data set using linear regression analysis; all model $F$ values are significant $(P \leq 0.0092)$. All parameter estimates are significant $(P \leq 0.0384)$, except for $b_{0}$ in $2008(P=0.0779)$.

${ }^{\mathrm{d}}$ Scientific E notation (e.g., E05 $=\times 10^{5}$ and E-4 $=\times 10^{-4}$ ).

${ }^{\mathrm{e}} R_{\mathrm{a}}{ }^{2}$ is the coefficient of determination adjusted for the number of independent variables.

TABLE 3. Regression analysis results from modeling sporulation of Fusicladium carpophilum on nectarine twig lesions as a function of temperature and duration of high relative humidity ${ }^{\mathrm{a}}$

\begin{tabular}{|c|c|c|c|c|c|c|c|c|c|c|c|}
\hline \multirow[b]{2}{*}{ Dependent variable, model } & \multicolumn{2}{|c|}{ Error } & \multicolumn{4}{|c|}{ Parameter estimate $^{\mathrm{b}}$} & \multicolumn{5}{|c|}{ Parameter CV $(\%)^{\mathrm{c}}$} \\
\hline & df & $\mathrm{MS}^{\mathrm{d}}$ & $m$ & $B_{0}$ & $B_{1}$ & $B_{2}$ & $m$ & $B_{0}$ & $B_{1}$ & $B_{2}$ & $\mathrm{CV}_{\mathrm{T}}$ \\
\hline \multicolumn{12}{|l|}{ Conidia/lesion, $\mathrm{S}_{1}$} \\
\hline Combined & 164 & 9.59Е04 & 0.8776 & 0.0201 & 4.91E-3 & $-1.10 \mathrm{E}-4$ & 2.1 & 26.4 & 12.4 & 14.5 & 55.4 \\
\hline \multicolumn{12}{|l|}{ Conidia/centimeter of twig, $\mathrm{S}_{2}$} \\
\hline Combined & 164 & $5.32 \mathrm{E} 06$ & 0.8524 & 0.0215 & $4.34 \mathrm{E}-3$ & $-9.00 \mathrm{E}-5$ & 2.3 & 30.2 & 17.2 & 22.2 & 71.9 \\
\hline Validation & 128 & $5.14 \mathrm{E} 06$ & 0.8813 & 0.0201 & $5.01 \mathrm{E}-3$ & $-1.10 \mathrm{E}-4$ & 2.4 & 37.3 & 16.9 & 20.0 & 76.6 \\
\hline
\end{tabular}

${ }^{a}$ Data were calculated from pooled observations on 10 twigs per treatment combination of temperature and duration of high relative humidity. Combined model derived from all treatment observations across all 3 years of the study $(n=168)$. The validation model was derived from a 132-observation subset of the combined data set; 44 observations were randomly chosen from each year of the study.

${ }^{\mathrm{b}}$ Richards model (equation 6) fit to each data set using nonlinear regression, where duration of high relative humidity represents incubation time, the rate $r$ is a quadratic function of temperature, and the upper asymptote $K$ is the maximum mean value observed.

${ }^{\mathrm{c}}$ Parameter coefficient of variation $(\mathrm{CV})=($ standard error/estimate $) \times 100 ; \mathrm{CV}_{\mathrm{T}}=$ total or sum of variation across all parameters.

${ }^{\mathrm{d}}$ Scientific E notation (e.g., E05 $=\times 10^{5}$ and E-3 $=\times 10^{-3}$ ). 
value for the validation model. Total parameter variation, $\mathrm{CV}_{\mathrm{T}}$, was slightly higher for the validation models, mostly due to an increase in variability when estimating the $b_{0}$ parameter.

The validation model data set had three fewer maximum sporulation values than the combined data set. The resulting Gaussian validation models, fit to this subset of maximum values, had parameter values that were slightly higher than those observed for the combined data models (Table 1). Total parameter variation, $\mathrm{CV}_{\mathrm{T}}$, was slightly lower for the validation models.

The relationship between the observed test data and predicted values of the validation model was linear $(P<0.0001)$, with 94 and $97 \%$ of the predicted values within the $95 \%$ prediction interval for the $S_{1}$ - and $S_{2}$-dependent variables, respectively (Fig. 4; Supplementary Figure 4). Furthermore, the slopes of the fitted association lines were not significantly different from one $(P=$ 0.92 and 0.24 for $S_{1}$ and $S_{2}$, respectively), and their intercepts were not significantly different from zero $(P=0.98$ and 0.48 for $\mathrm{S}_{1}$ and $\mathrm{S}_{2}$, respectively). These results indicate a near one-to-one correspondence between the predicted and observed data. However, a few outliers at high sporulation levels indicate that some improvement can be made in model predictions.

\section{DISCUSSION}

Sporulation of $F$. carpophilum on overwintering, 1-year-old nectarine twig lesions was quantified as a function of ambient air temperature and duration of $\mathrm{RH}>95 \%$. The production of conidia over time, when incubated at high atmospheric moisture, was mathematically characterized by a version of the Richards function, with properties intermediate between the monomolecular and Gompertz models (28). The final estimates of $m=0.85$ to 0.91 would dictate a more Gompertz-like model in which the absolute rate of sporulation $(d S / d t)$ rises rapidly at early stages of incubation but, once achieving its maximum value, then slowly declines toward zero (9). That is, relative to the symmetric logistic model, early spore production is faster. This mathematical behavior has obvious biological advantages for the pathogen be-

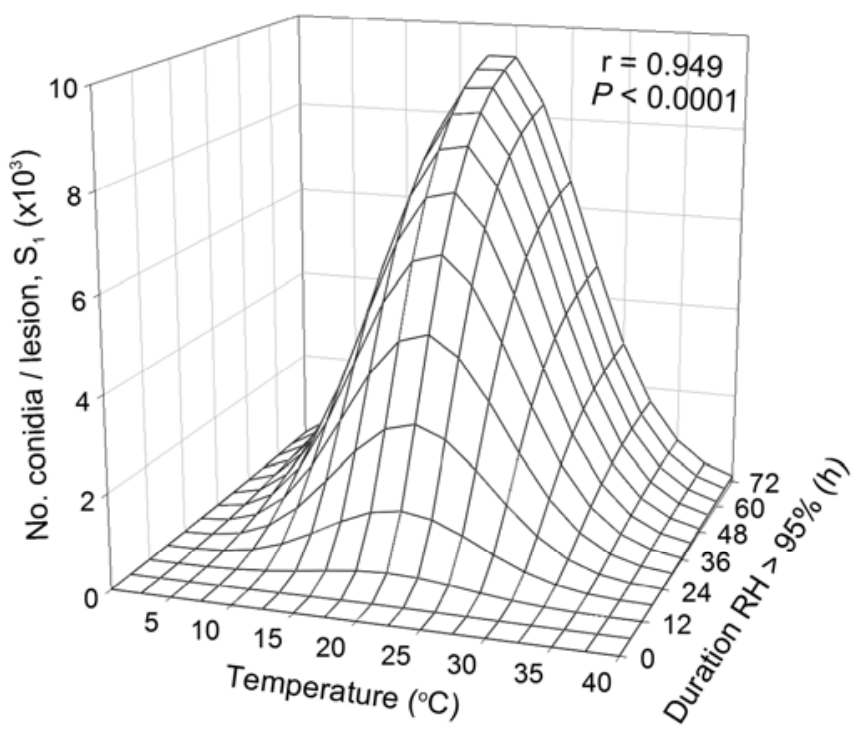

Fig. 3. Production of conidia by Fusicladium carpophilum lesions on 1-yearold nectarine twigs as a function of temperature and duration of high relative humidity. The three-dimensional response surface for number of conidia per lesion $\left(\mathrm{S}_{1}\right)$ consists of predicted data generated by the final, nonlinear form of the Richards model and its associated parameter functions (equation 9); the predicted curve was extrapolated beyond $35^{\circ} \mathrm{C}$ to complete visualization of the surface. Coefficients for the models were derived from the pooled 2007, 2008, and 2009 data sets (observations on 1,680 twigs). Pearson correlation coefficients $(r)$ were calculated to measure the degree of association between the predicted data and observed levels of sporulation. cause it minimizes the time required to produce large numbers of conidia at the start of a high RH period.

Earlier histological studies support the ability of the pathogen to quickly produce many conidia $(10,11)$. During the period between twig infection in the first year and sporulation in the second year, the pathogen was observed to amass considerable amounts of subcuticular stroma beneath the lesion surface. The presence of these stromatic masses, sometimes six to eight cells thick (10), most likely allows the pathogen to quickly produce large numbers of conidiophores and conidia in response to favorable conditions, thereby resulting in a rapid exponential rise indicative of the Gompertz model. In comparison, histological observations of twig lesions in their first summer of development showed much less subcuticular mycelium and only sparse formation of conidiophores and conidia under favorable conditions (10).

The effect of temperature on in vivo sporulation of F. carpophilum has not been previously investigated. In our study, the optima for sporulation on twig lesions, which were estimated by fitting the Gaussian model to the spore production maxima at each temperature, were 17.9 to $20.2^{\circ} \mathrm{C}$, with an overall average of $18.8^{\circ} \mathrm{C}$. In contrast, prior studies showed that in vitro growth of mycelium on various media was optimum at 20 to $27^{\circ} \mathrm{C}$ (10) and 20 to $25^{\circ} \mathrm{C}$ (19). This difference in optima for $F$. carpophilum is consistent with the generalization that most fungi have lower temperature optima for sporulation than for vegetative growth (4). Furthermore, the sporulation optima are very close in value to air temperatures typically observed in the southern New Jersey peach-growing region. During May and June, when twig lesion sporulation capacity is at its greatest (22), average monthly air temperatures at the RAREC for the 5-year period of 2006 to 2010 were 17.1 and $22.1^{\circ} \mathrm{C}$, respectively. The similarity of these temperature data indicates that the seasonal sporulation capacity of twig lesions is well synchronized with climatic conditions favorable to individual sporulation events.

Reaction rates for most biological processes, which are typically governed by enzymes, are highly dependent on tempera-

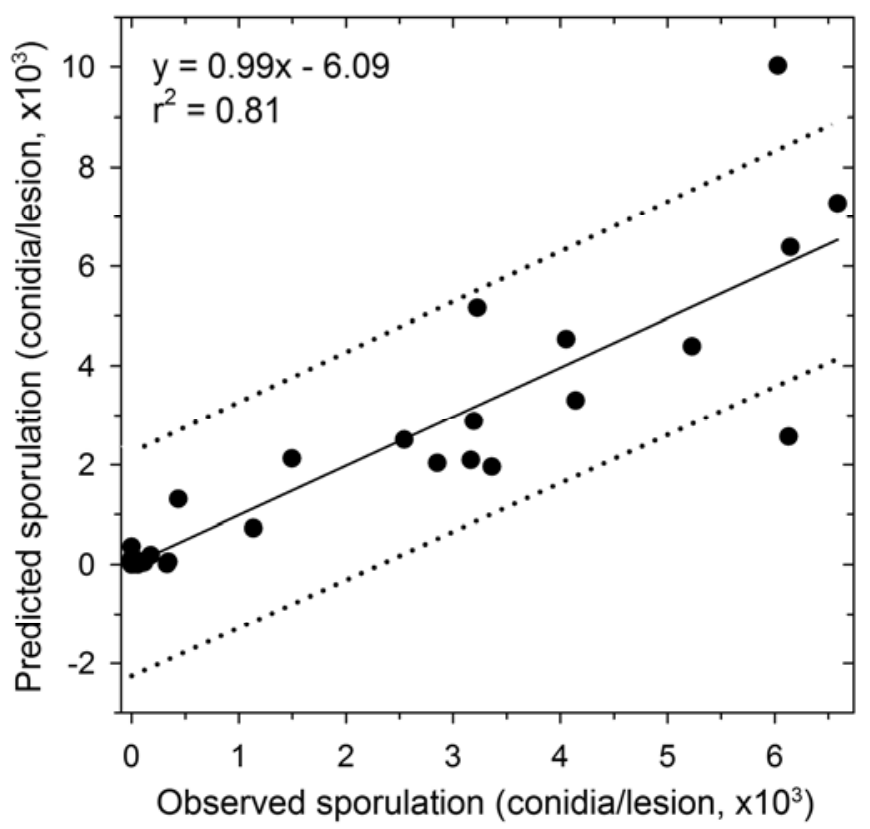

Fig. 4. Relationship between observed and predicted sporulation for the $S_{1}$ dependent variable (number of conidia per lesion). Predicted values were generated by the validation model (equation 9) created from a randomly chosen majority subset of the combined data; observed values were those data excluded from validation model development. The solid line and equation represent the linear association between observed and predicted data; dashed lines represent the $95 \%$ prediction interval. 
ture (20). In our model, the occurrence of sporulation required high RH (by definition) but, once this moisture prerequisite was achieved, the rate of sporulation was determined by temperature. In general, the reaction rate-temperature curve is characterized by a steady exponential increase at temperatures below the optimum, followed by an abrupt decrease above the optimum (20). Such curves often characterize the effect of temperature on the photosynthetic rate of plants (2). Essentially, rising temperatures increase reaction rates until denaturation of one or more enzymes causes rapid decline. Our results showed a similar response for the rate of sporulation of $F$. carpophilum when expressed as the Richards rate parameter. In particular, once the maximum sporulation rate was achieved at the optimum temperature, the decline occurred fairly quickly between 30 and $35^{\circ} \mathrm{C}$. Furthermore, spores produced at these high temperatures were somewhat elongated relative to those at cooler temperatures, which may be indicative of a dysfunctional physiology. Additional studies are needed to confirm this temperature-dependent morphological change and determine whether it affects viability.

An empirical equation, which utilizes key points readily determined from most data such as optimum and lethal temperatures, was developed to provide the necessary asymmetrical curve form for describing temperature-rate relationships $(24,25)$. When this model was fit to our Richards rate data, an excellent fit was obtained at the higher temperatures but a poor fit occurred at suboptimum temperatures (not shown). In comparison, the quadratic model provided a much better overall fit and, therefore, was utilized in the final sporulation model. Quadratic models were also found to provide good fits for sporulation rates of Plasmopara viticola on grape (15) and Phomopsis amygdali on peach (14). In reality, polynomials are frequently used to model temperature effects on biological processes because theoretical equations, typically based on the response of a single enzyme to temperature, often fit poorly to data describing organismal processes such as sporulation (30). Nevertheless, to avoid errant predictions of $F$. carpophilum sporulation, the proposed model should only be employed within the temperature range investigated; namely, 1 to $35^{\circ} \mathrm{C}$. Fortunately, this rather wide temperature range should encompass the vast majority of individual sporulation events occurring during the peak sporulation period in spring and early summer.

Spore production levels observed in 2009 were lower than those observed in the prior 2 years. This difference was particularly evident when comparing the amplitude parameters of the Gaussian model. Such variability would be expected for number of conidia per unit twig length because lesion density, which is dependent on infection conditions during the prior season, could vary from year to year. However, expression of the dependent variable as number of conidia per lesion did not correct for differences in lesion density. The higher numbers of lesions in 2009 resulted in fewer numbers of spores per lesion than in the previous 2 years. This outcome implied that the spore productionlesion density relationship was not linear at high levels of disease severity; that is, the capacity of the twigs to support pathogen growth and reproduction became limiting. However, because the 2009 lesion numbers were only $9.5 \%$ greater than those in 2007 , overall tree health may also have played an important role. By 2009, the orchard had not received fungicide applications for 3 years, and many heavily infected twigs did not survive the winter. Other variables, such as lesion size and age, may have also played a role in influencing spore production in each year.

The models developed in this study can be used for both simulation and forecasting purposes. In the latter case, sporulation events could be predicted for the purposes of timing fungicide applications. However, because this would be a sporulation-based system, forecasts would only indicate the risk of infection based on predicted amounts of available inoculum, as opposed to forecasting actual infection events. This type of forecasting sys- tem, based on a spore production and release model, was used successfully to time fungicide applications for onion leaf blight $(12,13)$; however, recent research has shown that actual measurement of airborne inoculum provides more accurate predictions (3). In the peach scab pathosystem, measurement of rain-splashed F. carpophilum conidia is certainly possible (18); however, an overall estimate of the number of overwintering twig lesions in a given orchard may be sufficient to scale inoculum potential accordingly. In addition, a spore survival model could be incorporated so that only viable conidia constitute the current available "inoculum load". This approach was utilized for a grape downy mildew model, which provided real-time output on viable inoculum levels based on forecasts from integrated sporulation and spore viability models (21). Finally, for a more holistic peach scab forecasting system, components that predict seasonal twig lesion sporulation capacity, spore dissemination, and infection risk could also be incorporated $(6,16,27,29)$.

The validation performed in this study compared predictions of models developed from a majority subset of the data with observations from the remaining "independent" data set. Because this comparison was performed using data chosen from each of the 3 years, the validation tested the overall effects of seasonal variability on both suspect and pathogen, particularly lesion growth and development. However, the incubation conditions for the sporulation of the twig lesions remained constant across all 3 years. Obviously, temperature and RH conditions can vary considerably during natural sporulation periods. Also, the capacity of lesions to sporulate on detached twigs could be different from their ability to sporulate on trees. Thus, to complete the validation process, model predictions need to be compared with sporulation observations from twig lesions in situ. In addition, sporulation data should be obtained from a variety of peach and nectarine cultivars to test the robustness of the model's predictions.

\section{ACKNOWLEDGMENTS}

This research was funded by the United States Department of Agriculture Cooperative State Research, Education, and Extension Service Northeast Regional IPM Competitive Grants Program (2007-3410318077) and by the New Jersey Agricultural Experiment Station. We thank R. Seem for his review of the manuscript prior to submission.

\section{LITERATURE CITED}

1. Anderson, H. W. 1956. Diseases of Fruit Crops. McGraw-Hill Book Co., New York.

2. Campbell, G. S. 1977. An Introduction to Environmental Biophysics. Springer-Verlag, New York.

3. Carisse, O., McRoberts, N., and Brodeur, L. 2008. Comparison of monitoring- and weather-based risk indicators of Botrytis leaf blight of onion and determination of action thresholds. Can. J. Plant Pathol. 30:442-456.

4. Carlile, M. J., and Watkinson, S. C. 1996. The Fungi. Academic Press, Harcourt Brace \& Company, London.

5. Cook, M. J., and Scherm, H. 1999. Antisporulant activity of early-season fungicide sprays against peach scab, 1998. Fungic. Nematicide Tests 54:53.

6. Gottwald, T. R. 1983. Factors affecting spore liberation by Cladosporium carpophilum. Phytopathology 73:1500-1505.

7. Hendrix, F. F., Jr. 1995. Scab. Pages 11-12 in: Compendium of Stone Fruit Diseases. J. M. Ogawa, E. I. Zehr, G. W. Bird, D. F. Ritchie, K. Uriu, and J. K. Uyemoto, eds. The American Phytopathological Society, St. Paul, MN.

8. Hirst, J. M., and Schein, R. D. 1965. Terminology of infection processes. Phytopathology 55:1157.

9. Jowett, D., Browning, J. A., and Cournoyer Haning, B. 1974. Nonlinear disease progress curves. Pages 115-136 in: Epidemics of Plant Diseases, Mathematical Analysis and Modeling. J. Kranz, ed. Springer-Verlag, New York.

10. Keitt, G. W. 1917. Peach scab and its control. U. S. Dep. Agric. Bull. 395.

11. Koch, L. W. 1934. Studies on the overwintering of certain fungi parasitic and saprophytic on fruit trees. Can. J. Res. 11:190-206.

12. Lacy, M. L. 1985. Timing onion leaf blight sprays with a dedicated 
disease predictor in Michigan. (Abstr.) Phytopathology 75:1284.

13. Lacy, M. L., and Pontius, G. A. 1983. Prediction of weather-mediated release of conidia of Botrytis squamosa from onion leaves in the field. Phytopathology 73:670-676.

14. Lalancette, N., Foster, K. A., and Robison, D. M. 2003. Quantitative models for describing temperature and moisture effects on sporulation of Phomopsis amygdali on peach. Phytopathology 93:1165-1172.

15. Lalancette, N., Madden, L. V., and Ellis, M. A. 1988. A quantitative model for describing the sporulation of Plasmopara viticola on grape leaves. Phytopathology 78:1316-1321.

16. Lalancette, N., McFarland, K. A., and Burnett, A. L. 2008. Reduction in primary inoculum of Fusicladosporium carpophilum on stone fruit by the QoI fungicide trifloxystrobin. (Abstr.) Phytopathology 98:S207.

17. Lalancette, N., McFarland, K. A., and Burnett, A. L. 2009. Modeling sporulation of Fusicladosporium carpophilum on nectarine twig lesions. (Abstr.) Phytopathology 99:S68.

18. Lan, Z., and Scherm, H. 2003. Moisture sources in relation to conidial dissemination and infection by Cladosporium carpophilum within peach canopies. Phytopathology 93:1581-1586.

19. Lawrence, E. G., and Zehr, E. I. 1982. Environmental effects on the development and dissemination of Cladosporium carpophilum on peach. Phytopathology 72:773-776.

20. Lehninger, A. L. 1975. Biochemistry, The Molecular Basis of Cell Structure and Function, 2nd ed. Worth Publishers, New York.

21. Madden, L. V., Ellis, M. A., Lalancette, N., Hughs, G., and Wilson, L. L. 2000. Evaluation of a disease warning system for downy mildew of grapes. Plant Dis. 84:549-554

22. Murday, E., Lalancette, N., and Foster, K. A. 2006. Anti-sporulant activity of trifloxystrobin on nectarine scab twig lesions. (Abstr.) Phytopathology 96:S181.

23. Neter, J., Wasserman, W., and Kutner, M. H. 1983. Applied Linear Regression Models. Richard D. Irwin, Inc., Homewood, IL.

24. O'Neill, R. V. 1968. Population energetics of a milipede, Narceus americanus (Beavois). Ecology 49:803-809.

25. O’Neill, R. V., Goldstein, R. A., Shugart, H. H., and Makin, J. B. 1972. Terrestrial ecosystem energy model. East. Deciduous For. Biome Memo Rep. No. 72-19.

26. Partridge, E. C., and Morgan-Jones, G. 2003. Notes on Hyphomycetes. XC. Fusicladisporum, a new genus for Cladosporium-like anamorphs of Venturia and the pecan scab-inducing fungus. Mycotaxon 85:357-370.

27. Pineau, R., Raymondaud, H., and Schiavon, M. 1991. Élaboration d'un modèle de prévision des risques d'infection du mirabellier (Prunus domestica L. var. insititia) par l'agent de la tavelure (Cladosporium carpophilum Thümen). Agronomie 11:561-570.

28. Richards, F. J. 1959. A flexible growth function for empirical use. J. Exp. Bot. 10:290-300.

29. Scherm, H., Savelle, A. T., Boozer, R. T., and Foshee, W. G. 2008. Seasonal dynamics of conidial production potential of Fusicladium carpophilum on twig lesions in southeastern peach orchards. Plant Dis. 92:47-50.

30. Spain, J. D. 1982. Basic Microcomputer Models in Biology. AddisonWesley Publishing Co., Reading, MA 